\title{
\begin{tabular}{l|l} 
Mibraries & DSpace@MIT
\end{tabular}
}

\author{
MIT Open Access Articles
}

\section{The Impact of Carrier Delocalization and Interfacial Electric Field Fluctuations on Organic Photovoltaics}

The MIT Faculty has made this article openly available. Please share how this access benefits you. Your story matters.

Citation: Kohn, Alexander W. et al. "The Impact of Carrier Delocalization and Interfacial Electric Field Fluctuations on Organic Photovoltaics." The Journal of Physical Chemistry C 121, 48 (November 2017): 26629-26636 (c) 2017 American Chemical Society

As Published: https://pubs.acs.org/doi/10.1021/acs.jpcc.7b08726

Publisher: American Chemical Society (ACS)

Persistent URL: http://hdl.handle.net/1721.1/115096

Version: Author's final manuscript: final author's manuscript post peer review, without publisher's formatting or copy editing

Terms of Use: Article is made available in accordance with the publisher's policy and may be subject to US copyright law. Please refer to the publisher's site for terms of use. 


\title{
The Impact of Carrier Delocalization and
}

\section{Interfacial Electric Field Fluctuations on Organic Photovoltaics}

\author{
Alexander W. Kohn, David P. McMahon, Shuhao Wen, and Troy Van Voorhis* \\ Department of Chemistry, Massachusetts Institute of Technology, Cambridge,
} Massachusetts 02139-4307

E-mail: tvan@mit.edu

\begin{abstract}
Organic photovoltaic (OPV) devices hold a great deal of promise for the emerging solar market. However, to unlock this promise it is necessary to understand how they generate free charges. Here, we analyze the energetics and charge delocalization of the interfacial charges in poly $\left(p\right.$-phenylene vinylene) $(\mathrm{PPV}) /[6,6]$-phenyl- $\mathrm{C}_{6} 1$-butyric acid methyl ester (PCBM) and poly(3-hexylthiophene-2,5-diyl) (P3HT) / PCBM devices. We find in the PPV system that the interface does not produce molecular disorder, but that an interfacial electric field is formed upon the inclusion of environmental polarization that promotes charge separation. In contrast, the P3HT system shows a significant driving force for charge separation due to interfacial disorder confining the hole. However, this feature is overpowered by the polarization of the electronic environment, which generates a field inhibiting charge separation. In the two systems studied herein, electrostatic effects dominate charge separation, overpowering interfacially-induced disorder. This suggests that when balancing polymeric order with electrostratic effects, the latter should take priority.
\end{abstract}




\section{Introduction}

Photovoltaic materials show great promise in helping us overcome challenges in power generation, having played an increasingly important role in the energy market over the last decade. ${ }^{1,2}$ Organic photovoltaic (OPV) technology in particular has attracted research attention in the past few decades due to its superior tunability and higher theoretical efficiency compared to traditional silicon-based solar cells. In recent years, efficiencies in excess of $10 \%$ have been achieved. ${ }^{2-4}$ However, improvement is necessary if OPVs are to compete with silicon. ${ }^{5}$ To effect this improvement we need a better understanding of how OPVs operate on the molecular level.

In solar cells employing inorganic semiconductors, absorption of photons generates weaklybound charge carriers due to the large dielectric constant of the medium. ${ }^{6}$ However, OPVs have a much smaller dielectric constant, thus the electron and hole are coulombically bound together as they cannot be screened enough to separate - this forms an exciton. In order to generate charge carriers in these systems a driving force must be produced to separate the exciton. One common device structure, the bulk heterojunction (BHJ), induces separation using an interface between two different materials, usually a polymeric electron-donor phase that is thought to host most of the excitons and a fullerene-based electron-acceptor phase. ${ }^{7}$ Because exciton diffusion lengths in OPVs are short, it is necessary to have the interface near to where the excitons are generated. ${ }^{8,9}$ Due to this constraint, BHJs are constructed with the two domains interpenetrating one another in order to maximize the interfacial surface area and provide excitons with the shortest possible path for charge separation. ${ }^{10}$ At the interface between the materials, the highest occupied molecular orbital of the donor and the lowest unoccupied molecular orbital of the acceptor have different energies. ${ }^{7}$ This difference drives the exciton to separate into charges and determines the open circuit voltage of the OPV device. ${ }^{11}$ Simple calculations of the binding energy of the charge-transfer (CT) state at the interface suggest that charge separation requires around $0.2-0.3 \mathrm{eV}$, a prohibitively high

barrier at room temperature. ${ }^{12}$ Despite this problem, experiments show near-unity interfacial 
charge separation efficiencies in many OPVs. ${ }^{13-15}$

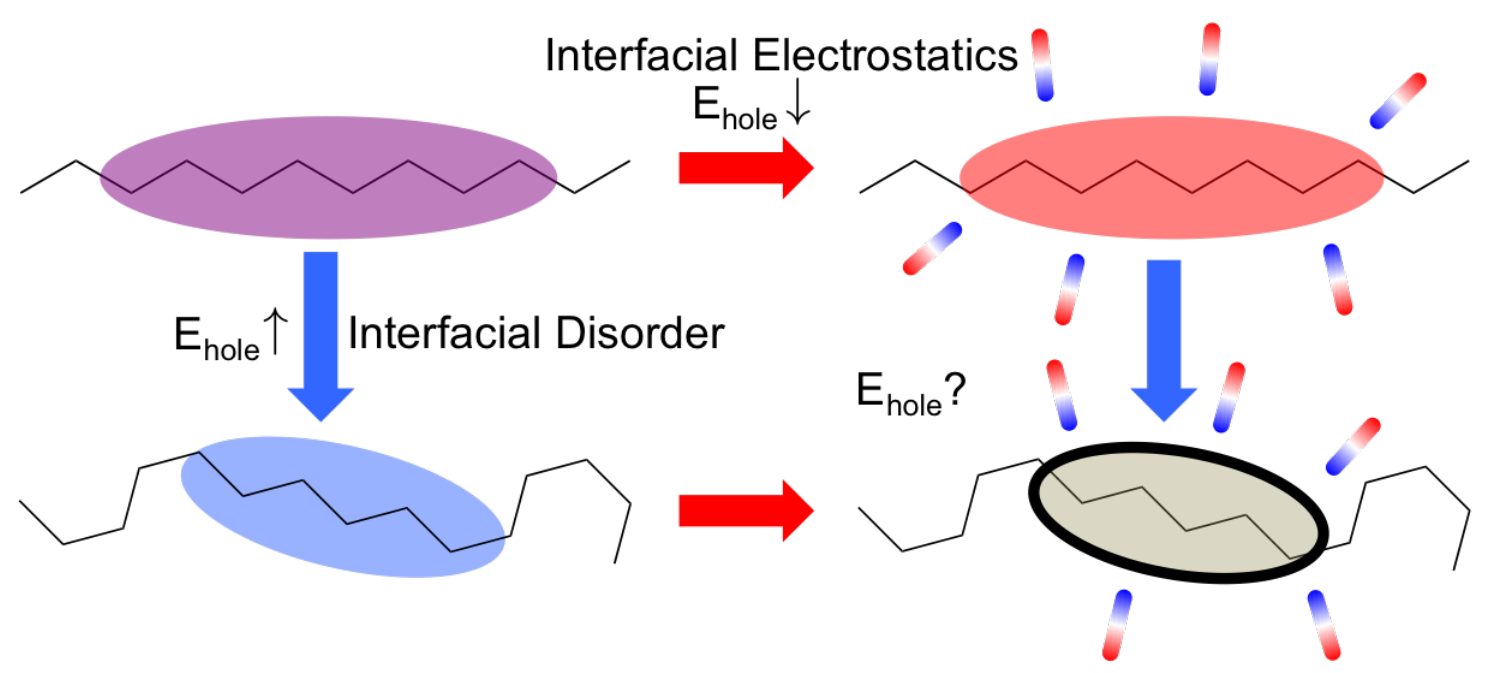

Figure 1: Schematic representing current state of studies on interfacial hole states in polymerfullerene BHJ devices. The upper left diagram represents the hole state of an ordered polymer in vacuo. Some studies have investigated and elaborated on the role of the electronic environment near the interface (top right). ${ }^{16-18}$ Others have examined disorder of the polymer chain and how that impacts the energetics and spatial extent of the hole state (bottom left). ${ }^{19,20}$ Herein, we examine both in a realistic system in order to evaluate how the two balance one another (bottom right).

We wish to address here the mystery over why charges separate at interfaces, which has generated controversy in the field. Some have suggested that this may be explained by invoking 'hot' high-energy CT states, in which excess energy from the charge-transfer process is used to overcome the charge binding energy. ${ }^{21-23}$ Ab initio calculations testing this hypothesis show that an electronically and vibrationally excited 'hot' CT state can indeed undergo facile interfacial charge separation. ${ }^{24,25}$ However, to date there is mixed experimental evidence for the necessity of 'hot' states. There is evidence that a larger donoracceptor offset is correlated with more efficient charge separation, ${ }^{23,26,27}$ suggesting that the energy released by the charge transfer event effects charge separation. Recent work has shown that one can directly probe optically-accessible CT states and found that for these states, the charge separation efficiency is independent of excitation energy. ${ }^{18,28-31}$ That is, 'hot' CT states and 'cold' CT states are equally efficient at dissociating. Others have criticized this interpretation, pointing out that these optically-excited CT states have not fully relaxed and 

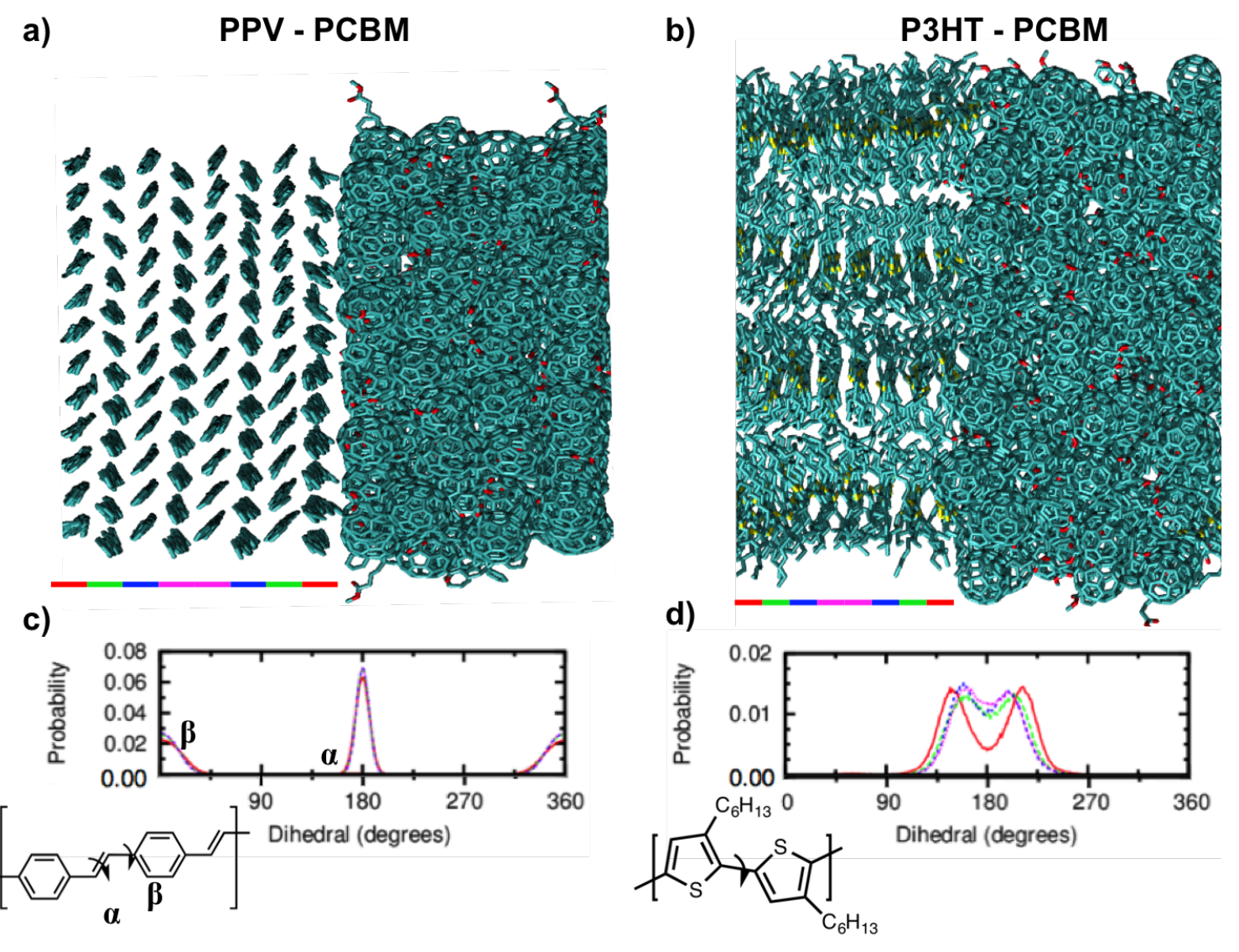

Figure 2: Images of the interfaces of (a) PPV/PCBM and (b) P3H PCBM and their dihedral angle distributions, (c) and (d) respectively. The dihedral angle distributions are separated based on their distance from the PCBM interface - red for the closest layers, purple for the farthest. 
that this polaronic stabilization energy is substantial. ${ }^{32}$

Generally speaking, there are two broad theoretical explanations for the apparent 'cold' CT state separation. The first posits that the electrostatic properties of the interface cause the band structures of the donor and acceptor molecules to bend such that there exists an energetic gradient that separates the charges. Various mechanisms have been suggested: the dielectric mismatch of the organic semiconductors; ${ }^{17,18}$ poor packing of molecules near the interface; ${ }^{19,20}$ and the presence of a static electric field near the interface. ${ }^{16}$

The second explanation focuses on the role of delocalization of the charges in the chargeseparated state. ${ }^{18,33,34}$ Due to the polymeric nature of the donor, the hole state can delocalize quite easily, decreasing its coulombic interaction with the electron and reducing the holeelectron binding energy. ${ }^{12,17,19}$ Other studies have implicated delocalization of the electron across multiple fullerene units in the acceptor phase. ${ }^{32,35,36}$

To understand the band structure near the interface, it is desirable to perform electronic structure calculations on representative systems. Unfortunately, BHJs are very disordered near the interface, and the exact nature of this disorder has a large impact on the performance of the device. ${ }^{37,38}$ Because of this, it is necessary to sample an ensemble of physically realistic states, mandating the use of molecular dynamics (MD). Previous studies have used MD to generate states for simulating the band structure of P3HT, but have not examined the combined effects of disorder and electronic polarization. ${ }^{39,40}$

Herein, we build on previous work suggesting that electronic polarization effects are important by incorporating Drude particles into our MD simulation and analyzing their impact on the interfacial electronic states. ${ }^{20,41,42}$ Our simulations include both realistic disorder in the molecular structures and the impact of the variable electrostatic environment near the interface, as summarized in Figure 1. We find that these polarization effects are necessary to reproduce the experimental band gap for the bulk species and have a substantial effect on the band structure near the interface. To probe the effect of disorder on charge carrier delocalization and band gaps, we examine interfaces with a relatively crys- 
talline donor phase $(\operatorname{poly}(p$-phenylene vinylene, PPV) and a relatively amorphous donor phase (poly(3-hexylthiophene-2,5-diyl), P3HT). We find that in PPV there is indeed little interfacial disorder, and that the interfacial band structure is dominated by a static electric field generated upon the inclusion of polarization effects. P3HT shows interfacial disorderinduced localization that promotes charge separation, but the effect is overpowered once electronic polarization effects are included. Taken together, these results imply that electronic polarization should take precedence over polymeric ordering when rationally designing the interfacial band structure of BHJ devices.

\section{Computational Details}

We examine two systems here: the PPV/ [6,6]-phenyl- $\mathrm{C}_{6} 1$-butyric acid methyl ester (PCBM) interface and the P3HT/PCBM interface. These two interfaces were chosen because PPV and P3HT are both well-characterized experimentally and show contrasting degrees of crystallinity, ${ }^{43,44}$ thus allowing us to probe the effect of molecular ordering on the band structure. ${ }^{45}$ There is some controversy in the literature regarding how crystallinity impacts BHJ efficiency, as there seems to be a tradeoff where increased crystallinity improves charge mobilities but decreases interfacial charge separation. ${ }^{46-51}$ We wish to address some of the ways that increased crystallinity may affect charge separation here.

The interfaces we model here are not perfect representations of the interfaces that appear in devices. Our simulations employ sharp interfaces between the donor and acceptor phases. However, under some processing conditions miscibility between the donor and acceptor phases is observed and an amorphous mixed phase seems to be present. ${ }^{52}$ Another difference is that the PPV we simulate lacks side chains, whereas experimentalists commonly use PPV derivatized bulky side chains that substantially impact crystallinity and interfacial structure. ${ }^{53}$ Use of our data to understand in situ experimental interfaces should bear these caveats in mind. 


\section{Molecular Dynamics}

The PPV-PCBM interface employed a crystalline slab of 96 PPV molecules abutting 240 PCBM molecules. Each PPV oligomer contained 14 monomer units, with the crystalline phase arranged into 8 layers of 12 chains. The starting PPV configuration was taken from from an experimental crystal structure ${ }^{54}$ and the force field was adapted from the work of DuBay and coworkers,${ }^{55}$ with point-charges taken from the central monomer of a B3LYP/6$31 \mathrm{G}^{* *}$ CHELPG calculation on a trimer.

The P3HT-PCBM interface simulations were performed with 32 P3HT molecules and 240 PCBM molecules (see Figure 2). For P3HT, the crystalline phase was composed of 20-mer chains organized into 4 layers, each consisting of 8 chains each, with the starting configuration developed as in reference. ${ }^{19}$ We chose this polymer length because the hole polaron in P3HT is thought to be delocalized over approximately 15 monomer units, allowing us to minimize the finite size effect on the energies. ${ }^{56}$ The majority of the force-field terms for P3HT are taken from previous studies, ${ }^{57,58}$ with the non-bonded terms (e.g., van der Waals terms) for all three systems (PPV, P3HT, and PCBM) coming from an optimized potentials for liquid simulations (OPLS) all-atom force field. ${ }^{59}$

The starting PCBM configuration came from an experimental crystal structure ${ }^{60}$ and consisted of 240 molecules. The force field was derived from previous studies. ${ }^{61,62}$

The initial P3HT, PPV and PCBM systems were equilibrated (with independent PCBM simulations run that had interfacial surface areas equal to each of the polymer systems). Configurations were first energy minimized, followed by a $1 \mathrm{~ns}$ NVT simulation at $100 \mathrm{~K}$, then with a $1 \mathrm{~ns}$ NPT simulation at $100 \mathrm{~K}$ and $300 \mathrm{~K}$ using a 1 fs timestep, 12 Angstrom cutoff with a potential shift for Lennard-Jones and short-range Coulombic interactions, the Berendsen thermostat, particle mesh Ewald for the long-range electrostatics, and an anisotropic barostat with relaxation times of 0.1 and $1.0 \mathrm{ps}$, respectively. The equilibrated systems were resized to have equal surface area and aligned adjacent to one another with a 3-4 Angstrom gap between them. An energy minimization was then performed to remove close contacts, 
followed by a 1 ns NVT run at $500 \mathrm{~K}$ in which the P3HT was held rigid and the PCBM allowed to move to wet the interface. This was followed by a $1 \mathrm{~ns}$ NPT run at $100 \mathrm{~K}$, and finally a $5 \mathrm{~ns}$ NPT run at $300 \mathrm{~K}$, the last 2 ns of which were used for subsequent analysis. Snapshots from these runs were taken every 40 ps in order to decrease the correlation between snapshots. All molecular dynamics simulations utilized Gromacs. ${ }^{63}$ Short-range interactions used a All system visualizations were performed with VMD. ${ }^{64}$ Forcefield parameters and the coordinates of a representative snapshot can be found in the Supporting Information (SI).

\section{Electronic Structure Calculations}

From a given MD snapshot, multiple molecules are chosen and separate quantum chemical calculations are performed on each one. We performed the calculations while treating the molecular environment in three ways: as absent (i.e., in vacuum); as point charges; or as point charges with corresponding Drude particles. Calculations in vacuo for these systems that incorporate atomistic disorder have been performed elsewhere in the literature (e.g., in work by Troisi $\left.{ }^{19}\right)$. However, treatments of the electrostatic environment have not been. Drude particles are useful in this context because they simulate electronic polarizability, whereas a point charge treatment alone does not respond dynamically to the electronic field of the interrogated molecule. We shall refer to these three levels of treating the environment as "Vacuum", "Static", and "Polarizable".

The Drude particles' locations in the chemical system are described in the supporting information (SI) and their parameters were optimized to reproduce the experimental dielectric

constant. All quantum chemical calculations were performed at the LRC-wPBEh/6-31g* level ${ }^{65}$ using QChem (version 4.1). ${ }^{66}$ This functional was chosen as it has been shown to describe the extent of delocalization in polymer chains well ${ }^{67,68}$ and employs $20 \%$ Hartree-Fock exchange and $\omega=0.2 \mathrm{bohr}^{-1}$. 


\section{Results and Discussion}

Table 1: Calculated transport properties for PCBM, PPV, and PCBM. The ionization potential (IP) and electron affinity (EA) have been calculated for the bulk phase of the three materials and with the three environmental treatments being considered. The transition gap (TG) is computed as $E_{\mathrm{IP}}-E_{\mathrm{EA}}$. Improved treatment of the environment brings theoretical values in line with experimental values. Each value has a standard deviation of $0.05 \mathrm{eV}$.

\begin{tabular}{lcccc}
\hline & & $\mathrm{IP}(\mathrm{eV})$ & $\mathrm{EA}(\mathrm{eV})$ & $\mathrm{TG}(\mathrm{eV})$ \\
\hline \hline \multirow{3}{*}{$\mathrm{PCBM}^{26}$} & Vacuum & 7.45 & 1.74 & 5.71 \\
$(\epsilon=3.9)$ & Static & 6.92 & 2.23 & 4.69 \\
& Polarizable & 6.25 & 3.43 & 2.82 \\
& Experimental & $5.9-6.1$ & 3.7 & 2.4 \\
\hline & Vacuum & 5.73 & 1.75 & 3.98 \\
$\mathrm{PPV}^{69}$ & Static & 5.51 & 1.85 & 3.66 \\
$(\epsilon=2.6)$ & Polarizable & 5.28 & 2.28 & 3.00 \\
& Experimental & 4.9 & 2.8 & 2.1 \\
\hline & Vacuum & 5.35 & 1.31 & 4.04 \\
$\mathrm{P} \mathrm{HT}^{26}$ & Static & 5.12 & 1.25 & 3.87 \\
$(\epsilon=3.4)$ & Polarizable & 4.91 & 2.11 & 2.79 \\
& Experimental & 4.8 & 2.2 & 2.6 \\
\hline
\end{tabular}

\section{Bulk Properties}

We parameterized the Drude particles to reproduce the bulk polarizabilities of PPV, P3HT, and PPV, as computed from the Clausius-Mossotti relation and the experimental dielectric constants. During this procedure, we calculated the bulk ionization potential (IP), electron affinity (EA), and transport gap (TG, $\left.E_{\mathrm{IP}}-E_{\mathrm{EA}}\right)$ for each organic semiconductor. These results are reported in Table 1.

For all three bulk systems, inclusion of environmental polarizability drastically improves the agreement of these quantities with their experimental values. For PCBM, Drude particles stabilize both charged states by around 0.7-0.8 eV, consistent with the Born model solvation energy for a charge in a spherical cavity of radius 7 Angstroms and continuum dielectric of $3.9(0.76 \mathrm{eV}) .{ }^{20,70}$ The two polymers display a similar behavior, but of a lesser magnitude. For PPV and P3HT, the ionized states stabilize by only $0.3 \mathrm{eV}$ and $0.4 \mathrm{eV}$ respectively. Even 

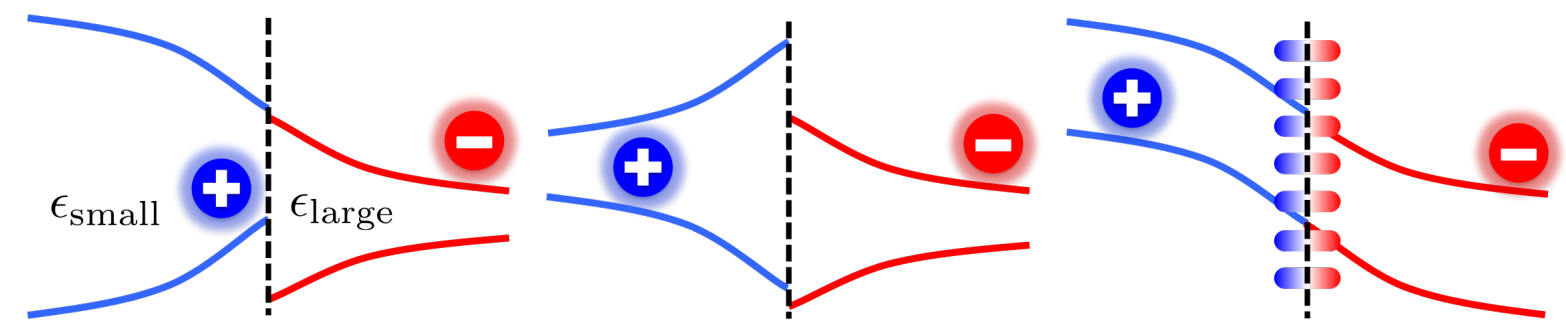

Figure 3: The impact of different interfacial contexts on the band structures. A dielectric mismatch in which the acceptor phase has a larger dielectric than the donor phase (left) causes the bands of the donor phase to become stabilized and the bands of the acceptor phase to become destabilized as the hole and electron become better and worse solvated, respectively. Interfacial disorder (center) disrupts conjugation, confining and destabilizing the hole and electron states. A static interfacial electric field (right) stabilizes the electrons and destabilizes the holes in one domain (depicted here as the donor phase), and destabilizes the electrons and stabilizes the holes in the other.
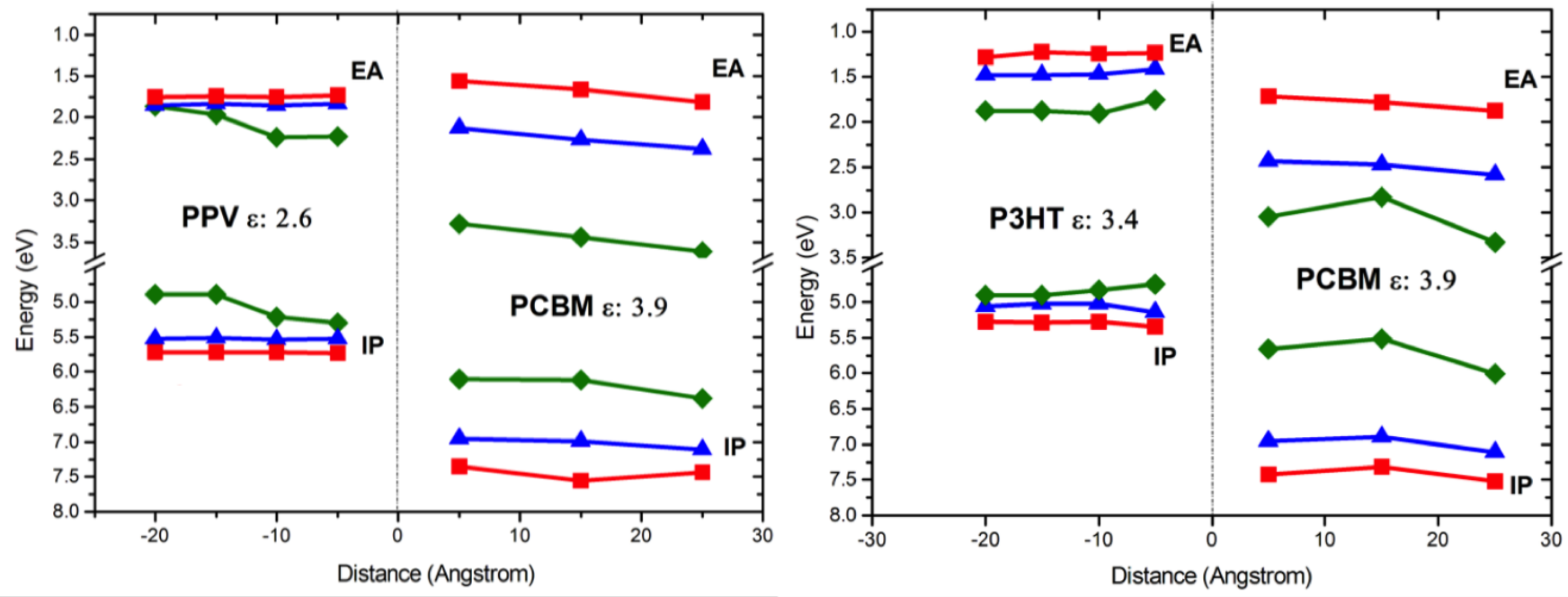

Figure 4: The band structures at the PPV-PBCM interface (left) and the P3HT-PCBM interface (right) with differing treatments of the environment: as vacuum (red); static point charges (blue); or as static point charges with Drude particles (green). In both systems, inclusion of polarization effects dramatically changes the band structure. Each point has been averaged from 102 calculations and has a standard deviation of $0.05 \mathrm{eV}$. The distances are resolved by binning each molecule into a layer and using the average distance from the interface of that layer. 
accounting for the polymeric systems' smaller dielectric constants, a Born model calculation would imply an unphysical cavity radius of tens of Angstroms. This suggests more forces are relevant than simply electrostatics - namely, a delocalization pressure. That is, as the dielectric increases, the size of the polaronic charge will tend to contract so as to maximize the interaction with the dielectric. This contraction will increase the kinetic energy of the hole, reducing the apparent stabilization energy. We can probe this interplay by modeling the electrons and holes as particles in a one-dimensional box solvated by the spherical born model:

$$
\mathrm{E}=-\left(1-\frac{1}{\epsilon}\right) \frac{1}{\mathrm{~L}}+\frac{\pi^{2}}{2 \mu \mathrm{L}^{2}}
$$

where $\epsilon$ is the relative dielectric of the medium, $\mathrm{L}$ is the diameter of the box, and $\mu$ is the effective mass of the pseudoparticle (which accounts in an approximate way for the deviation of the true potential from a particle in a box). At the equilibrium value of L ( 30 Angstroms for $\epsilon \approx 4$ ), this expression reduces to:

$$
\left.\mathrm{E}\right|_{\frac{\mathrm{dE}}{\mathrm{dL}}=0}=-\frac{\mu}{2 \pi}\left(1-\frac{1}{\epsilon}\right) \approx-1.38 \mu\left(1-\frac{1}{\epsilon}\right) \mathrm{eV}
$$

Typical effective mass values for holes and electrons in organic polymers are around 0.2 $\mathrm{m}_{\mathrm{e}} \cdot{ }^{71}$ When we evaluate the solvation energy by subtracting the energy of the unsolvated charge (with $\mathrm{L}=37$ Angstroms), we find stabilization energies for PPV and P3HT of 0.18 $\mathrm{eV}$ and $0.21 \mathrm{eV}$ (these numbers are sensitive to the exact value of $\mu$ used). These values are much closer to the numbers we computed, showing that this simple model can explain the lower stabilization energies.

\section{Interfacial Molecular Structure}

The constructed interfaces show starkly different levels of structural disorder, as can be seen visually in Figures 2a and 2b. In PPV the torsional angles have very tight distributions (Fig. 
2, part c), a feature which varies minimally as we approach the interface. This contrasts dramatically with the situation for P3HT (Fig. 2, part d), where we observe a broad dihedral distribution that separates significantly as we approach the interface. These results confirm what can be seen by visual inspection of the two interfaces: the P3HT/PCBM interface is more disordered than the PPV/P3HT interface. Additionally, these findings agree well with experimental studies of these systems ${ }^{45}$ wherein PPV is known to be much more structured than P3HT and reflects our use of PPV and P3HT as generic models of an inflexible and flexible donor, respectively.

\section{Interfacial Electronic Structure}

We evaluated the IP/EA levels of molecules as a function of distance from the interface in order to determine how the interface affects the energetics of charge separation (Fig.

4). Figure 3 shows how common interfacial phenomena impact the local band structure: ${ }^{20}$ a dielectric mismatch promotes the separation of one of the charged species to the bulk, interfacial disorder disrupts conjugation, confining and destabilizing the charge carriers, and a static interfacial electric field either works with or against separation for both charge carriers.

Turning to the PPV-PCBM interface (Fig. 4, left), we observe that the IP and EA levels of the polymeric donor phase are agnostic towards the interface unless we include electronic polarization effects. This suggests both that the high degree of crystallinity of PPV persists at the interface and that the static charge distribution of interfacial PCBM molecules has little impact, hence, the hole levels do not change as we approach PCBM. When we include electronic polarization effects, charge carriers in PPV display contrasting responses to the interface, with interfacial electrons becoming stabilized and interfacial holes relatively destabilized. This asymmetric interfacial shift (where the EA and IP levels both go down) implies that an electric field may be induced at the interface that is preferentially stabilizing the electron levels. We expected the dielectric mismatch between PPV $(\epsilon=2.6)$ 
and PCBM $(\epsilon=3.9)$ to stabilize the interfacial holes, but that effect appears to be negligible in our calculations. Because holes flow "uphill" (towards regions with lower IP), the topology of this band structure encourages charge separation. The PCBM bands in the PPV-PCBM interface appear to show some influence from the dielectric mismatch, with the band gap broadening slightly, and a larger effect from the inclusion of polarization bending both levels up.

For the P3HT-PCBM interface (Fig. 4, right), the levels without polarization effects show a broadening of the interfacial band gap consistent with a disordered interface. This disorder disrupts conjugation, raising the confinement energy - this lowers the interfacial EA and raises the interfacial IP, thereby providing an energetic pressure for charge separation. When electronic polarization effects are enabled, however, interfacial P3HT hole states are stabilized (the IP band bends upward), which could be explained by the higher dielectric of the acceptor phase (although the electron levels in P3HT are mostly unaffected, perhaps due to being localized on a different part of the polymer with a different electrostatic environment). The PCBM domain shows surprisingly non-monotonic behavior in its band levels that resists an easy explanation. Despite this, the transport gap is decreasing monotonically as we look at PCBM molecules farther away from the interface, a result that is well-explained by the lower dielectric of P3HT.

Reflecting on the competing influences of electrostatic environment and molecular disorder in determining the interfacial band structure, we observe in PPV that the interfacial disorder seems to have no effect on the energy levels of PPV, but that electronic polarization effects do create a significant pressure for charge separation. In contrast, P3HT shows the impact of disorder on the hole energies, as the gas phase calculations show a significant decrease of the hole energy levels near the interface, promoting charge separation. Nonetheless, this effect is washed out by inclusion of the electrostatic environment - in both systems environmental electrostatics dominate the interfacial band structure in a manner that promotes charge separation. 

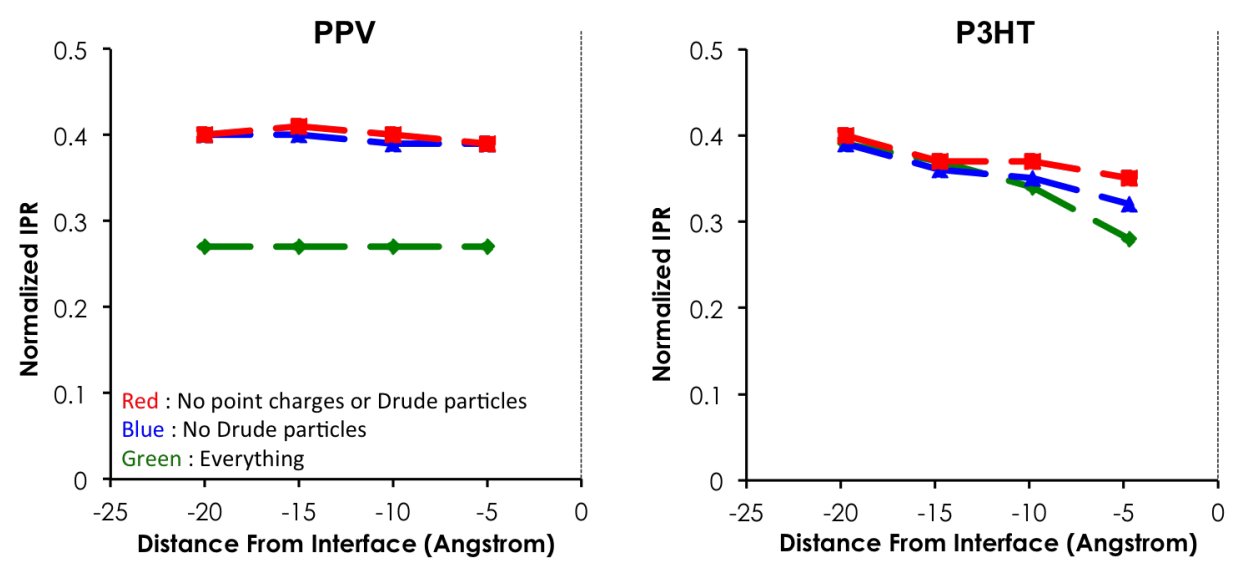

Figure 5: The normalized inverse participation ratios (NIPR) for the holes states in the electron-donating polymers being considered, PPV and P3HT. The NIPR was computed for each system with the environment treated as vacuum (red); static point charges (blue); and as static point charges with Drude particles (green). In PPV (left), the extent of delocalization of the hole states shows little influence from the interface, irregardless of the environmental treatment. In P3HT (right), the hole states are more localized near the interface than the bulk, with this effect strengthening as the environment is treated with greater sophistication. ach point has been averaged from 102 calculations and has a standard deviation of 0.01 . The distances are resolved by binning each molecule into a layer and using the average distance from the interface of that layer.

\section{Hole Delocalization}

In this study we are also interested in examining how the interface affects delocalization in the hole electronic states. One common method of doing this is by looking at the HOMO of the neutral polymer species. ${ }^{17,72,73}$ However, this measure neglects orbital relaxation, causing the hole to appear over-delocalized. Herein, we employ the normalized inverse participation ratio (NIPR), which counts the effective fraction of the polymer a hole state is spread over. We compute this as:

$$
\mathrm{NIPR}=\frac{1}{N} \frac{1}{\sum_{i \in \text { Sites }} \frac{1}{P_{i}}}
$$

Where $P_{i}$ is the population on each site of the hole state, which in this case is taken as the charge on each monomer within the polymer. This per-monomer population is computed as the difference in the Mulliken populations for the cationic and neutral forms of the polymer. 
The NIPR ranges between two extremes. For a model polymer in which the hole is equally distributed over $N$ sites, each with population $\frac{1}{N}, \mathrm{NIPR}=1$, the hole is distributed over the entire polymer. For a polymer in which the hole is entirely localized on 1 site, NIPR $=\frac{1}{N}$, reflecting the fact that the hole occupies only a small part of the polymer.

We compute the NIPR in the per-monomer basis instead of the per-atom basis because the per-atom basis results in sites with negative populations due to inductive effects and instabilities in the construction of Mulliken charges. The per-monomer treatment taken here has no negative-population sites, assuring us that the Mulliken populations used are reasonably accurate.

The average NIPR values for the hole states per layer are shown in Figure 5. NIPR data for the electron states in the polymers is in the SI.

As anticipated, PPV shows little sensitivity to the interface - the NIPR does not change as we approach the interface. The P3HT analysis shows a very different situation. Hole states in P3HT that are nearer to the interface are more localized. This arises from interfaciallyinduced disorder that causes a form of Anderson localization and has been observed in theoretical studies on P3HT in vacuo. ${ }^{44,74}$

As we compare different levels of environmental electrostatics, some interesting results accrue. For PPV, adding static point charges has a negligible effect - the NIPR remains constant and shows that the hole is localized on around $40 \%$ of the polymer. Drude particles dramatically localize the hole state to around two-thirds of the prior extent. In contrast, P3HT shows a change of a much smaller magnitude. Improved treatment of the electronic environment increases the degree of interfacial localization, but at most this only reduces the hole size around 20\%. The starkly different responses of these two materials to Drude particles suggests that the hole delocalization of disordered materials may be well-described without resorting to use of a polarizable environment, but this result may not be general.

These results are in concordance with our earlier interpretation of the band diagrams in Figure 4. PPV shows little structural impact from the interface, with the hole being equally 
delocalized near the interface and far away. There is, however, a significant dielectric con-

finement effect, wherein the $\frac{1}{\mathrm{~L}}$ dependence on the Born solvation energy squeezes the hole, increasing its kinetic energy. As inferred above, P3HT's conjugation is disrupted near the interface, causing the observed destabilization of interfacial holes in the gas phase calculations. Although dielectric confinement effects increase the magnitude of the interfacial localization as observed in Figure 5, the expected destabilization of interfacial holes does not appear in the band structure, as the impact of this structural disorder on the holes is washed out by the improved dielectric solvation of the PCBM domain.

\section{Conclusion}

In this study we analyze the impact of electrostatic effects and realistic disorder in simulations of BHJ interfaces. As we treat the environment with more sophistication, we observe that the band structure near the interface changes. The band gap narrows considerably for all molecules under consideration and substantial dependence on the interfacial distance develops due to interactions with a physically-realistic polarizable environment. For PPV, the interface causes little disorder, so that the final interfacial band shape emerges entirely from environmental electrostatics and shows a substantial pressure for charge separation. In P3HT, the interfacial chains are more disordered, localizing the hole states and raising their energies. The impact on the band structure, however, disappears when we account for the environment.

With regards to the controversy over whether electrostatic or delocalization effects are primary in understanding charge separation, what we observe herein suggests that interfacial disorder can indeed significantly lower the extent of the hole wavefunction. This effect is augmented by the inclusion of a sophisticated environment, but the environment itself also has an effect on the interfacial band structure that goes in the opposites direction of, and typically is stronger than, interfacial localization. 
When one considers OPV device design, there are many competing heuristics to balance. The results here suggest that one should give precedence to electrostatic environment considerations over interfacial disorder considerations when optimizing BHJ performance. Indeed, recent experimental devices incorporating a high-dielectric dopant into BHJs have found that despite this dopant introducing disorder into the system, the dielectric effect dominates and can be used to improve efficiencies. ${ }^{75}$

Future work dedicated to understanding how these effects manifests themselves in novel high-efficiency BHJ devices such as PTB7-PCBM would be of value. ${ }^{76}$ Additionally, much of the discussion on using charge delocalization to understand CT state separation is focused on delocalization in the acceptor domain between multiple fullerenes. ${ }^{24,32}$ Given our access to a realistically-disordered fullerene phase with a polarizable environment, such an investigation would be quite feasible. Finally, the systems we investigate here are still very ordered, with both the donor and acceptor phases merely perturbed from their crystalline geometries. A more realistically disordered system is less amenable to analysis, but would more completely describe the disorder found in real systems.

\section{Supporting Information}

Details on the construction of the Drude models, the IPR values, density of states plots, and forcefield parameters.

\section{Acknowledgements}

This work was funded by ENI S.p.A. as part of the MIT Energy Initiative. We thank Nadav Geva, Dr. James Shepherd, and Dr. Michael Mavros for helpful discussions. 


\section{References}

(1) Forrest, S. R. The path to ubiquitous and low-cost organic electronic appliances on plastic. Nature 2004, 428, 911-918.

(2) Li, G.; Zhu, R.; Yang, Y. Polymer solar cells. Nature Photonics 2012, 6, 153-161.

(3) Hu, H.; Jiang, K.; Yang, G.; Liu, J.; Li, Z.; Lin, H.; Liu, Y.; Zhao, J.; Zhang, J.;

Huang, F. et al. Terthiophene-based D-A polymer with an asymmetric arrangement of alkyl chains that enables efficient polymer solar cells. Journal of the American Chemical Society 2015, 137, 14149-14157.

(4) Chen, J. D.; Cui, C.; Li, Y. Q.; Zhou, L.; Ou, Q. D.; Li, C.; Li, Y.; Tang, J. X. Single-junction polymer solar cells exceeding $10 \%$ power conversion efficiency. Advanced Materials 2015, 27, 1035-1041.

(5) Dou, L.; You, J.; Hong, Z.; Xu, Z.; Li, G.; Street, R. A.; Yang, Y. 25th anniversary article: A decade of organic/polymeric photovoltaic research. Advanced Materials 2013, 25, 6642-6671.

(6) Nelson, J. The Physics of Solar Cells. Properties of Semiconductor Materials 2003, 384.

(7) Deibel, C.; Dyakonov, V. Polymer-Fullerene Bulk Heterojunction Solar Cells. Rep. Prog. Phys. 2010, 73, 1-39.

(8) Halls, J. J. M.; Walsh, C. A.; Greenham, N. C.; Marseglia, E. A.; Friend, R. H.; Moratti, S. C.; Holmes, A. B. Efficient photodiodes from interpenetrating polymer networks. 1995.

(9) Yu, G.; Gao, J.; Hummelen, J. C.; Wudl, F.; Heeger, A. J. Polymer Photovoltaic Cells: Enhanced Efficiencies via a Network of Internal Donor-Acceptor Heterojunctions. Science 1995, 270, 1789-1791. 
(10) Yang, X.; Loos, J.; Veenstra, S. C.; Verhees, W. J. H.; Wienk, M. M.; Kroon, J. M.; Michels, M. A. J.; Janssen, R. A. J. Nanoscale morphology of high-performance polymer solar cells. Nano Letters 2005, 5, 579-583.

(11) Rand, B. P.; Burk, D. P.; Forrest, S. R. Offset energies at organic semiconductor heterojunctions and their influence on the open-circuit voltage of thin-film solar cells. Physical Review B - Condensed Matter and Materials Physics 2007, 75, 1-11.

(12) Zhu, X. Y.; Yang, Q.; Muntwiler, M. Charge-transfer excitons at organic semiconductor surfaces and interfaces. Accounts of Chemical Research 2009, 42, 1779-1787.

(13) Park, S. H.; Leclerc, M.; Heeger, A. J.; Lee, K. High efficiency polymer solar cells with internal quantum efficiency approaching 100\%. Proceedings of SPIE 2009, 7416, 74160P-74160P-10.

(14) Few, S.; Frost, J. M.; Nelson, J. Models of charge pair generation in organic solar cells. Phys. Chem. Chem. Phys. 2015, 17, 2311-2325.

(15) Kippelen, B.; Brédas, J.-L. Organic photovoltaics. Energy 83 Environmental Science 2009, 2, 251.

(16) Cornil, J.; Verlaak, S.; Martinelli, N.; Mityashin, A.; Olivier, Y.; Van Regemorter, T.; D’Avino, G.; Muccioli, L.; Zannoni, C.; Castet, F. et al. Exploring the energy landscape of the charge transport levels in organic semiconductors at the molecular scale. Accounts of Chemical Research 2013, 46, 434-443.

(17) Castet, F.; D’Avino, G.; Muccioli, L.; Cornil, J.; Beljonne, D. Charge separation energetics at organic heterojunctions: on the role of structural and electrostatic disorder. Phys. Chem. Chem. Phys. 2014, 16, 20279-20290.

(18) Bässler, H.; Köhler, A. Hot or cold: how do charge transfer states at the donoracceptor 
interface of an organic solar cell dissociate? Phys. Chem. Chem. Phys. 2015, 17, 2845128462.

(19) McMahon, D. P.; Cheung, D. L.; Troisi, A. Why holes and electrons separate so well in polymer/fullerene photovoltaic cells. Journal of Physical Chemistry Letters 2011, 2, $2737-2741$.

(20) Yost, S. R.; Van Voorhis, T. Electrostatic effects at organic semiconductor interfaces: A mechanism for "cold" exciton breakup. Journal of Physical Chemistry C 2013, 117, $5617-5625$.

(21) Bakulin, A. A.; Dimitrov, S. D.; Rao, A.; Chow, P. C. Y.; Nielsen, C. B.; Schroeder, B. C.; McCulloch, I.; Bakker, H. J.; Durrant, J. R.; Friend, R. H. Chargetransfer state dynamics following hole and electron transfer in organic photovoltaic devices. Journal of Physical Chemistry Letters 2013, 4, 209-215.

(22) Dimitrov, S. D.; Bakulin, A. a.; Nielsen, C. B.; Schroeder, B. C.; Du, J.; Bronstein, H.; Mcculloch, I.; Friend, R. H.; Durrant, J. R.; Schroeder, B. C. On the Energetic Dependence of Charge Separation in Low Bandgap Polymer / Fullerene Blends On the Energetic Dependence of Charge Separation in Low Bandgap Polymer / Fullerene Blends. Journal of the American Chemical Society 2012, 10-13.

(23) Jailaubekov, A. E.; Willard, A. P.; Tritsch, J. R.; Chan, W.-L.; Sai, N.; Gearba, R.; Kaake, L. G.; Williams, K. J.; Leung, K.; Rossky, P. J. et al. Hot charge-transfer excitons set the time limit for charge separation at donor/acceptor interfaces in organic photovoltaics. Nature Materials 2012, 12, 66-73.

(24) Tamura, H.; Burghardt, I. Ultrafast charge separation in organic photovoltaics enhanced by charge delocalization and vibronically hot exciton dissociation. Journal of the American Chemical Society 2013, 135, 16364-16367. 
(25) Shen, X.; Han, G.; Fan, D.; Xie, Y.; Yi, Y. Hot charge-transfer states determine exciton dissociation in the DTDCTB $/ \mathrm{C}<$ inf $>60</$ inf $>$ complex for organic solar cells: A theoretical insight. Journal of Physical Chemistry C 2015, 119, 11320-11326.

(26) Ohkita, H.; Cook, S.; Astuti, Y.; Duffy, W.; Tierney, S.; Zhang, W.; Heeney, M.; McCulloch, I.; Nelson, J.; Bradley, D. D. et al. Charge carrier formation in polythiophene/fullerene blend films studied by transient absorption spectroscopy. Journal of the American Chemical Society 2008, 130, 3030-3042.

(27) Dimitrov, S. D.; Durrant, J. R. Materials design considerations for charge generation in organic solar cells. Chemistry of Materials 2014, 26, 616-630.

(28) Lee, J.; Vandewal, K.; Yost, S. R.; Bahlke, M. E.; Goris, L.; Baldo, M. A.; Manca, J. V.; Voorhis, T. V. Charge transfer state versus hot exciton dissociation in polymer-fullerene blended solar cells. Journal of the American Chemical Society 2010, 132, 11878-11880.

(29) Vandewal, K.; Albrecht, S.; Hoke, E. T.; Graham, K. R.; Widmer, J.; Douglas, J. D.; Schubert, M.; Mateker, W. R.; Bloking, J. T.; Burkhard, G. F. et al. Efficient charge generation by relaxed charge-transfer states at organic interfaces. Nature Materials 2013, 13, 63-68.

(30) Gautam, B. R.; Younts, R.; Li, W.; Yan, L.; Danilov, E.; Klump, E.; Constantinou, I.; So, F.; You, W.; Ade, H. et al. Charge Photogeneration in Organic Photovoltaics: Role of Hot versus Cold Charge-Transfer Excitons. Advanced Energy Materials 2016, 6, 1-6.

(31) Brédas, J. L.; Norton, J. E.; Cornil, J.; Coropceanu, V. Molecular understanding of organic solar cells: The challenges. Accounts of Chemical Research 2009, 42, 16911699.

(32) Savoie, B. M.; Rao, A.; Bakulin, A. A.; Gelinas, S.; Movaghar, B.; Friend, R. H.; Marks, T. J.; Ratner, M. A. Unequal partnership: Asymmetric roles of polymeric donor 
and fullerene acceptor in generating free charge. Journal of the American Chemical Society 2014, 136, 2876-2884.

(33) Nayak, P. K.; Narasimhan, K. L.; Cahen, D. Separating charges at organic interfaces: Effects of disorder, hot states, and electric field. Journal of Physical Chemistry Letters 2013, 4, 1707-1717.

(34) Pandit, B.; Jackson, N. E.; Zheng, T.; Fauvell, T. J.; Manley, E. F.; Orr, M.; BrownXu, S.; Yu, L.; Chen, L. X. Molecular Structure Controlled Transitions between FreeCharge Generation and Trap Formation in a Conjugated Copolymer Series. Journal of Physical Chemistry C 2016, 120, 4189-4198.

(35) Jamieson, F. C.; Domingo, E. B.; McCarthy-Ward, T.; Heeney, M.; Stingelin, N.; Durrant, J. R. Fullerenecrystallisation as a key driver of charge separation in polymer/fullerene bulk heterojunction solar cells. Chem. Sci. 2012, 3, 485-492.

(36) Lakhwani, G.; Rao, A.; Friend, R. H. Bimolecular Recombination in Organic Photovoltaics. Annual Review of Physical Chemistry 2014, 65, 557-581.

(37) Peumans, P.; Uchida, S.; Forrest, S. R. Efficient bulk heterojunction photovoltaic cells using small-molecular-weight organic thin films. Nature 2003, 425, 158-162.

(38) Buchaca-Domingo, E.; Vandewal, K.; Fei, Z.; Watkins, S. E.; Scholes, F. H.; Bannock, J. H.; De Mello, J. C.; Richter, L. J.; DeLongchamp, D. M.; Amassian, A. et al. Direct correlation of charge transfer absorption with molecular donor:acceptor interfacial area via photothermal deflection spectroscopy. Journal of the American Chemical Society 2015, 137, 5256-5259.

(39) Liu, T.; Cheung, D. L.; Troisi, A. Structural variability and dynamics of the P3HT/PCBM interface and its effects on the electronic structure and the chargetransfer rates in solar cells. Phys. Chem. Chem. Phys. 2011, 13, 21461-21470. 
(40) Huix-Rotllant, M.; Tamura, H.; Burghardt, I. Concurrent effects of delocalization and internal conversion tune charge separation at regioregular polythiophene-fullerene heterojunctions. Journal of Physical Chemistry Letters 2015, 6, 1702-1708.

(41) Difley, S.; Wang, L. P.; Yeganeh, S.; Yost, S. R.; Voorhis, T. V. Electronic properties of disordered organic semiconductors via QM/MM simulations. Accounts of Chemical Research 2010, 43, 995-1004.

(42) Yost, S. R.; Wang, L. P.; Van Voorhis, T. Molecular insight into the energy levels at the organic donor/acceptor interface: A quantum mechanics/molecular mechanics study. Journal of Physical Chemistry C 2011, 115, 14431-14436.

(43) Hoppe, H.; Sariciftci, N. S. Morphology of polymer/fullerene bulk heterojunction solar cells. J. Mater. Chem. 2006, 16, 45-61.

(44) McMahon, D. P.; Troisi, A. Organic semiconductors: Impact of disorder at different timescales. ChemPhysChem 2010, 11, 2067-2074.

(45) Grozema, F. C.; Van Duijnen, P. T.; Berlin, Y. A.; Ratner, M. A.; Siebbeles, L. D. Intramolecular charge transport along isolated chains of conjugated polymers: Effect of torsional disorder and polymerization defects. Journal of Physical Chemistry B 2002, $106,7791-7795$.

(46) Schrader, M.; Körner, C.; Elschner, C.; Andrienko, D. Charge transport in amorphous and smectic mesophases of dicyanovinyl-substituted oligothiophenes. Journal of Materials Chemistry 2012, 22, 22258.

(47) Poelking, C.; Andrienko, D. Effect of polymorphism, regioregularity and paracrystallinity on charge transport in poly(3-hexylthiophene) [P3HT] nanofibers. Macromolecules 2013, 46, 8941-8956. 
(48) Kordt, P.; Van Der Holst, J. J. M.; Al Helwi, M.; Kowalsky, W.; May, F.; Badinski, A.; Lennartz, C.; Andrienko, D. Modeling of Organic Light Emitting Diodes: From Molecular to Device Properties. Advanced Functional Materials 2015, 25, 1955-1971.

(49) Laquai, F.; Andrienko, D.; Mauer, R.; Blom, P. W. M. Charge carrier transport and photogeneration in P3HT:PCBM photovoltaic blends. Macromolecular Rapid Communications 2015, 36, 1001-1025.

(50) Kim, K. H.; Park, S.; Yu, H.; Kang, H.; Song, I.; Oh, J. H.; Kim, B. J. Determining optimal crystallinity of diketopyrrolopyrrole-based terpolymers for highly efficient polymer solar cells and transistors. Chemistry of Materials 2014, 26, 6963-6970.

(51) Woo, C. H.; Thompson, B. C.; Kim, B. J.; Toney, M. F.; Fréchet, J. M. J. The influence of poly(3-hexylthiophene) regioregularity on fullerene-composite solar cell performance. Journal of the American Chemical Society 2008, 130, 16324-16329.

(52) Collins, B. A.; Tumbleston, J. R.; Ade, H. Miscibility, crystallinity, and phase development in P3HT/PCBM solar cells: Toward an enlightened understanding of device morphology and stability. Journal of Physical Chemistry Letters 2011, 2, 3135-3145.

(53) Tajima, K.; Suzuki, Y.; Hashimoto, K. Polymer Photovoltaic Devices Using Fully Regioregular Poly[(2-methoxy-5-(3,7-dimethyloctyloxy))-1,4-phenylenevinylene]. The Journal of Physical Chemistry C 2008, 112, 8507-8510.

(54) Granier, T.; Thomas, E. L.; Gagnon, D. R.; Karasz, F. E.; Lenz, R. W. Structure investigation of poly(pphenylene vinylene). Journal of Polymer Science Part B: Polymer Physics 1986, 24, 2793-2804.

(55) Dubay, K. H.; Hall, M. L.; Hughes, T. F.; Wu, C.; Reichman, D. R.; Friesner, R. A. Accurate force field development for modeling conjugated polymers. Journal of Chemical Theory and Computation 2012, 8, 4556-4569. 
(56) Niklas, J.; Mardis, K. L.; Banks, B. P.; Grooms, G. M.; Sperlich, A.; Dyakonov, V.; Beaupré, S.; Leclerc, M.; Xu, T.; Yu, L. et al. Highly-efficient charge separation and polaron delocalization in polymerfullerene bulk-heterojunctions: a comparative multifrequency EPR and DFT study. Physical Chemistry Chemical Physics 2013, 15, 9562.

(57) Moreno, M.; Casalegno, M.; Raos, G.; Meille, S. V.; Po, R. Molecular modeling of crystalline alkylthiophene oligomers and polymers. Journal of Physical Chemistry B 2010, 114, 1591-1602.

(58) Arosio, P.; Moreno, M.; Famulari, A.; Raos, G.; Catellani, M.; Meille, S. V. Ordered stacking of regioregular head-to-tail polyalkylthiophenes: Insights from the crystal structure of form I' poly(3-n-butylthiophene). Chemistry of Materials 2009, 21, 78-87.

(59) Jorgensen, W. L.; Maxwell, D. S.; Tirado-Rives, J. Development and testing of the OPLS all-atom force field on conformational energetics and properties of organic liquids. Journal of the American Chemical Society 1996, 118, 11225-11236.

(60) Rispens, M. T.; Meetsma, A.; Rittberger, R.; Brabec, C. J.; Sariciftci, N. S.; Hummelen, J. C. Influence of the solvent on the crystal structure of PCBM and the efficiency of MDMO-PPV:PCBM 'plastic' solar cells. Chemical communications (Cambridge, England) 2003, 80, 2116-8.

(61) Cheung, D. L.; Troisi, A. Theoretical Study of the Organic Photovoltaic Electron Acceptor PCBM: Morphology, Electronic Structure, and Charge Localization $<\sup ></ \sup >$. The Journal of Physical Chemistry C 2010, 114, 20479-20488.

(62) Huang, D. M.; Faller, R.; Do, K.; Moule, A. J. Coarse-grained computer simulations of polymer/fullerene bulk heterojunctions for organic photovoltaic applications. Journal of Chemical Theory and Computation 2010, 6, 526-537.

(63) Hess, B.; Kutzner, C.; van der Spoel, D.; Lindahl, E. GROMACS 4: algorithms for 
highly efficient, load balanced, and scalable molecular simulations. J. Chem. Theory and Comput. 2008, 4, 435-447.

(64) Humphrey, W.; Dalke, A.; Schulten, K. \{\} : \{Visual $\}$ molecular dynamics. Journal of Molecular Graphics 1996, 14, 33-38.

(65) Rohrdanz, M. A.; Martins, K. M.; Herbert, J. M. A long-range-corrected density functional that performs well for both ground-state properties and TD-DFT excitation energies, including charge-transfer excited states. Journal of Chemical Physics 2009, $130,054112$.

(66) Shao, Y.; Al, E. Advances in molecular quantum chemistry contained in the Q-Chem 4 program package. Molecular Physics 2015, 113, 184-215.

(67) Shao, N.; Wu, Q. Charge self-localization in $\pi$-conjugated polymers by long range corrected hybrid functionals. Physical Chemistry Chemical Physics 2014, 16, 6700.

(68) Nayyar, I. H.; Batista, E. R.; Tretiak, S.; Saxena, A.; Smith, D. L.; Martin, R. L. Localization of electronic excitations in conjugated polymers studied by DFT. Journal of Physical Chemistry Letters 2011, 2, 566-571.

(69) Greenwald, Y.; Xu, X.; Fourmigue, M.; Srdanov, G.; Koss, C.; Wudl, F.; Heeger, a. J. Polymer-polymer rectifying heterojunction based on poly(3,4-dicyanothiophene) and MEH-PPV. Journal of Polymer Science Part a-Polymer Chemistry 1998, 36, 31153120.

(70) Born, M. Volume and hydration warmth of ions. Zeitschrift Fur Physik 1920, 1, 45-48.

(71) Hutchison, G. R.; Zhao, Y.-J.; Delley, B.; Freeman, A. J.; Ratner, M. A.; Marks, T. J. Electronic structure of conducting polymers:Limitations of oligomer extrapolation approximations and effects of heteroatoms. Physical Review B 2003, 68, 035204. 
(72) Cheung, D. L.; McMahon, D. P.; Troisi, A. A realistic description of the charge carrier wave function in microcrystalline polymer semiconductors. Journal of the American Chemical Society 2009, 131, 11179-11186.

(73) Qin, T.; Troisi, A. Relation between Structure and Electronic Properties of Amorphous MEH-PPV Polymers. J Am Chem Soc 2013, 135, 11247-11256.

(74) Barford, W.; Lidzey, D. G.; Makhov, D. V.; Meijer, A. J. Exciton localization in disordered poly(3-hexylthiophene). Journal of Chemical Physics 2010, 133, 1-7.

(75) Leblebici, S.; Lee, J.; Weber-Bargioni, A.; Ma, B. Dielectric Screening to Reduce Charge Transfer State Binding Energy in Organic Bulk Heterojunction Photovoltaics. Journal of Physical Chemistry C 2017, 121, 3279-3285.

(76) Liang, Y.; Xu, Z.; Xia, J.; Tsai, S. T.; Wu, Y.; Li, G.; Ray, C.; Yu, L. For the bright future-bulk heterojunction polymer solar cells with power conversion efficiency of $7.4 \%$. Advanced Materials 2010, 22, 135-138. 


\section{TOC Graphic}
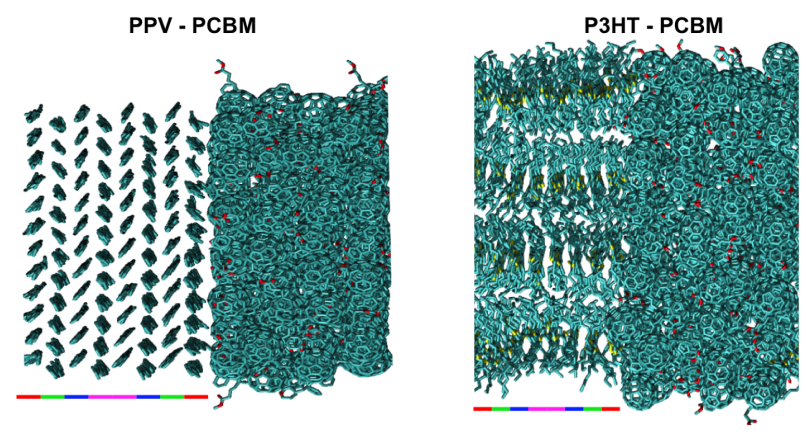